\title{
On the Construction of Preliminary Test Estimators of the Reliability Characteristics for the Exponential Distribution \\ Based on Records
}

\author{
Ajit Chaturvedi \\ Department of Statistics, University of Delhi, Delhi - 110007, INDIA \\ Email: ajitc2001@yahoo.co.in \\ Ananya Malhotra \\ Department of Statistics, University of Delhi, Delhi - 110007, INDIA \\ Corresponding Author Email: malhotra.ananya3@gmail.com
}

\begin{abstract}
Synoptic Abstract: The one-parameter exponential distribution plays an important role in reliability theory. Two measures of reliability for exponential distribution are considered, $R(t)=P(X>t)$ and $P=P(X>Y)$. Sometimes, due to past knowledge or experience, the experimenter may be in a position to make an initial guess on some of the parameters of interest. In such cases, we can provide an improved estimator by incorporating the prior information on the parameters. Preliminary test estimators (PTES) have been developed in the literature for the parameters of various distributions. To the best of the knowledge of the authors, PTES are not available for $R(t)$ and $P$. For record values from exponential distribution, we define PTES based on uniformly minimum variance unbiased estimator (UMVUE), maximum likelihood estimator (MLE) and empirical Bayes estimator (EBE) for the powers of the parameter, $R(t)$ and $P$. Bias and mean square error (MSE) expressions for the proposed estimators are derived. A comparative study of different methods of estimation is done through simulation and it is established that PTES perform better than ordinary UMVUES, MLES and EBES.
\end{abstract}

Keywords and Phrases: Exponential distribution; Empirical Bayes estimator; Preliminary test estimator; Record values; Monte Carlo Simulation

Mathematical Subject Classification 2010: Primary: 62N05; Secondary: 62F10, 62F03

\section{Introduction}


In statistical inference, many a times we come across problems where there may exist some known prior information on the parameters often regarded as constraints. Whenever we have some information available in the form of a point guess value on the parameters of the distribution, then in order to be sure that this information is valid, inferential estimation procedures can be developed to estimate those parameters by incorporating this prior information. This notion introduced the concept of preliminary test estimators to check the validity of our hypothesis on the parameter and also obtain more precise estimates. Bancroft (1944) introduced the use of PTES and eventually further advancements were proposed by Saleh and Sen (1978), Saleh and Kibria (1993), Kibria (2004), Saleh (2006), Kibria and Saleh (1993, 2004, 2005, 2006, 2010) and Belaghi, Arashi and Tabatabaey (2014, 2015). Until now in the literature of inferential statistics, the researchers have developed PTES of the parameters of different distributions. To the best of the knowledge of the authors, no preliminary test estimators have been developed for the reliability functions $R(t)$ and $P$ based on records. In the present paper, we derive PTES for two measures of reliability functions. The reliability function $R(t)$ is defined as the probability of failure-free operation until time $t$. Thus, if the random variable $(r v) X$ denotes the lifetime of an item or a system, then $R(t)=P(X>t)$. One may refer to Sinha (1986) for further reading. Another measure of reliability under stress-strength setup is the probability $P=P(X>Y)$, which represents the reliability of an item or a system of random strength $X$ subject to random stress $Y$. Kotz et al. (2003, p. 14) have discussed the theory and applications of the stress-strength relationships in industrial and economic systems.

A lot of work has been done in the literature for the point estimation and testing of $R(t)$ and $P$. For a brief review, one may refer to Pugh (1963), Basu (1964), Bartholomew (1957, 1963), Tong (1974, 1975), Johnson (1975), Kelley, Kelley and Schucany (1976), Sathe and Shah (1981), Chao (1982), Chaturvedi and Surinder (1999), Awad and Gharraf (1986), Tyagi and Bhattacharya (1989) and Chaturvedi and Rani (1997, 1998), Chaturvedi and Tomer (2002, 2003), Chaturvedi and Singh (2006, 2008), Chaturvedi and Kumari (2015), Chaturvedi and Malhotra (2016, 2017) and Chaturvedi and Pathak (2012, 2013, 2014).

Chandler (1952) introduced the concept of record values. Based on records, inferential procedures for the parameters of different distributions have been developed by Glick (1978), Nagaraja (1988a,1988b), Balakrishan, Ahsanullah and Chan (1995), Arnold, Balakrishan and Nagaraja (1992), Habibi Rad, Arghami and Ahmadi (2006), Arashi and Emadi (2008), Razmkhah and Ahmadi (2011) and others. 
The exponential distribution is one of the most widely used continuous distributions in reliability analysis. It is popularly used to model the time elapsed between events and to study the behaviour of items that have constant failure rate, i.e. items that do not wear out. Since this distribution has numerous remarkable properties, it has many characterisations of both theoretical and practical importance. Having just one parameter, this model is quite simple to elucidate and implement. For more uses of the exponential distribution, one may refer to Epstein and Sobel (1953).

Let the $r v X$ follow the exponential distribution with probability density function $(p d f)$, cumulative distribution function $(c d f)$ and reliability function at time point $t$ respectively given by:

$$
\begin{gathered}
f(x ; \theta)=\frac{1}{\theta} e^{\frac{-x}{\theta}} ; x \geq 0, \theta>0 \\
F(x ; \theta)=1-e^{\frac{-x}{\theta}} ; x \geq 0, \theta>0 \\
R(t)=e^{-\frac{t}{\theta}}
\end{gathered}
$$

In engineering, stress is a solid body (liquids do not admit engineering stress) which may arise due to some applied load and is defined as "the force per unit area that one part of the body exerts on its adjacent parts". Psychological stress is also another type of stress. If a $r v X$ follows exponential distribution with mean life $\theta_{1}$ and a $r v Y$ independent of $X$ is an exponential variable with mean life $\theta_{2}$, then the reliability function under stress-strength setup is obtained as

$$
P=\theta_{1}\left(\theta_{1}+\theta_{2}\right)^{-1}
$$

Let $X_{1}, X_{2}, \ldots$ be an infinite sequence of independent and identically distributed (iid) rvs with $f f(x ; \theta)$. An observation $X_{j}$ will be called an upper record value (or simply a record) if its value exceeds that of all previous observations. Thus $X_{j}$ is a record if $X_{j}>X_{i}$ for every $i<j$. The record time sequence $\left\{T_{n}, n \geq 0\right\}$ is defined as

$$
\left\{\begin{array}{c}
T_{0}=1 \quad ; \text { with probability } 1 \\
T_{n}=\min \left\{j: X_{j}>X_{T_{n-1}}\right\} ; n \geq 1
\end{array}\right.
$$

and the record value sequence $\left\{R_{n}\right\}$ is then defined as 


$$
R_{n}=X_{T_{n}} ; n=0,1,2, \ldots
$$

then the likelihood function of $\theta$ given the first $n+1$ upper record values $R_{0}, R_{1}, R_{2}, \ldots, R_{n}$ is:

$$
\begin{aligned}
L\left(\theta \mid R_{0}, R_{1}, R_{2}, \ldots, R_{n}\right) & =f\left(R_{n} ; \theta\right) \prod_{i=0}^{n-1} \frac{f\left(R_{i}, \theta\right)}{1-F\left(R_{i} ; \theta\right)} \\
& =\frac{1}{\theta^{n+1}} e^{\frac{-R n}{\theta}} .
\end{aligned}
$$

The rest of the paper is organised as follows. In Section 2, on the basis of records, we construct PTES based on MLE, UMVUE and EBE for the powers of the parameter of exponential distribution. We also propose PTES based on MLE and UMVUE of the reliability functions $R(t)$ and $P$ on the basis of record values. Then, bias and MSE expressions of the proposed estimators are obtained in Section 3. In Section 4, we study the relative efficiency of the proposed estimators over the usual estimators on the basis of simulated data and finally in Section 5, we discuss the results obtained.

\section{Proposed Preliminary Test Estimators}

Let $R_{0}, R_{1}, R_{2}, \ldots, R_{n}$ be the first $n+1$ upper record values from the distribution defined in (1). Then from Chaturvedi and Malhotra (2016), for $p \in(-\infty, \infty), p \neq 0$ the MLE of $\theta^{p}$ is:

$$
\hat{\theta}_{M L}^{p}=\left(\frac{R_{n}}{n+1}\right)^{p}
$$

where $R_{n}$ is the complete and sufficient statistic of $\theta$ and has gamma distribution with parameters $(n+1, \theta)$. Further, the UMVUE of $\theta^{p}$ is:

$$
\hat{\theta}_{U}^{p}=\frac{\Gamma(n+1)}{\Gamma(n+p+1)}\left(R_{n}\right)^{p}
$$

Now, if we consider conjugate prior distribution of $\theta$ to be Inverted-Gamma distribution with parameters $(\mu, v)$ and $p d f$ : 


$$
\pi(\theta)=\frac{\mu^{v}}{\Gamma(v) \theta^{v+1}} e^{\frac{-\mu}{\theta}} ; \mu, \theta>0 \text { and } v \text { is a positive integer, }
$$

then the posterior distribution of $\theta$ given $R_{0}, R_{1}, R_{2}, \ldots, R_{n}$ is:

$$
\pi\left(\theta \mid R_{0}, R_{1}, R_{2}, \ldots, R_{n}\right)=\frac{\left(R_{n}+\mu\right)^{n+v+1}}{\Gamma(n+v+1) \theta^{n+v+2}} e^{\frac{-\left(R_{n}+\mu\right)}{\theta}}
$$

Under squared error loss function, the Bayes estimator of $\theta^{p}$ is:

$$
\hat{\theta}_{B}^{p}=\frac{\Gamma(n+v-p+1)}{\Gamma(n+v+1)}\left(R_{n}+\mu\right)^{p} ; v>-(n-p+1)
$$

Also, the marginal distribution of $R_{0}, R_{1}, R_{2}, \ldots, R_{n}$ given $\mu$ and $v$ is:

$$
\begin{aligned}
m\left(R_{0}, R_{1}, R_{2}, \ldots, R_{n} \mid \mu, v\right) & =\int_{0}^{\infty} \pi(\theta) L\left(\theta \mid R_{0}, R_{1}, R_{2}, \ldots, R_{n}\right) d \theta \\
& =\frac{\mu^{v} \Gamma(n+v+1)}{\left(R_{n}+\mu\right)^{n+v+1} \Gamma(v)} .
\end{aligned}
$$

Taking the natural logarithm $l$ of the above marginal distribution, the MLE of $\mu$ and $v$ can be obtained from the solution of the following system:

$$
\frac{\partial l}{\partial \mu}=\frac{v}{\mu}-\frac{n+v+1}{R_{n}+\mu}=0
$$

and

$$
\frac{\partial l}{\partial v}=\log (\mu)+\frac{\partial}{\partial v}\left[\log \left(\frac{\Gamma(n+v+1)}{\Gamma(v)}\right)\right]-\log \left(R_{n}+\mu\right)=0
$$

Denoting the MLE of $\mu$ and $v$ by $\hat{\mu}_{M L}$ and $\hat{v}_{M L}$ respectively, then there exists a relation between them given by:

$$
\hat{\mu}_{M L}=\frac{R_{n} \hat{v}_{M L}}{n+1}
$$

Therefore, from (11), the $\mathrm{EBE}$ of $\theta^{p}$ is: 


$$
\hat{\theta}_{E B}^{p}=\frac{\Gamma\left(n+\hat{v}_{M L}-p+1\right)}{\Gamma\left(n+\hat{v}_{M L}+1\right)}\left(R_{n}+\hat{\mu}_{M L}\right)^{p} ; \hat{v}_{M L}>-(n-p+1) .
$$

It is worthwhile to mention that we are interested in the powers of the parameter $\theta$ as they can be utilised in estimating the moments of the exponential distribution.

In the sequel to the estimators defined in equations (7), (8) and (12), we define three different PTES based on MLE, UMVUE and EBE of $\theta^{p}$ when it is suspected that $\theta$ may be equal to $\theta_{o}$. Often the information on the value of $\theta$ is available from the past knowledge or experiments. This non-sample prior information can be expressed in the form of the following group of hypotheses:

$$
\begin{aligned}
& H_{o}: \theta=\theta_{o} \\
& H_{1}: \theta \neq \theta_{o},
\end{aligned}
$$

then based on classical hypothesis testing, the critical region is given by:

$$
\left\{0<R_{n}<k_{\mathrm{o}}\right\} \cup\left\{k_{\mathrm{o}}{ }^{\prime}<R_{n}<\infty\right\},
$$

where $k_{\mathrm{o}}$ and $k_{\mathrm{o}}{ }^{\prime}$ are obtained such that $k_{\mathrm{o}}=\frac{\theta_{\mathrm{o}}}{2} \chi_{2(n+1)}^{2}\left(\frac{\alpha}{2}\right), k_{\mathrm{o}}{ }^{\prime}=\frac{\theta_{\mathrm{o}}}{2} \chi_{2(n+1)}^{2}\left(1-\frac{\alpha}{2}\right)$ and $\alpha$ is the level of significance. Or, equivalently we reject $H_{o}$ if:

$$
\frac{2 R_{n}}{\theta_{o}}<C_{2} \text { or } \frac{2 R_{n}}{\theta_{o}}>C_{1},
$$

where $C_{1}=\chi_{2(n+1)}^{2}\left(1-\frac{\alpha}{2}\right)$ and $C_{2}=\chi_{2(n+1)}^{2}\left(\frac{\alpha}{2}\right)$.

Thus we define three PTES for $\theta^{p}$ based on MLE, UMVUE and EBE respectively as:

and

$$
\begin{aligned}
\hat{\theta}_{M L}^{p P T} & =\hat{\theta}_{M L}^{p}-\left(\hat{\theta}_{M L}^{p}-\theta_{o}^{p}\right) I(A), \\
\hat{\theta}_{U}^{p P T} & =\hat{\theta}_{U}^{p}-\left(\hat{\theta}_{U}^{p}-\theta_{o}^{p}\right) I(A)
\end{aligned}
$$

where $I(A)$ is the indicator function of the set:

$$
A=\left\{\chi_{2(n+1)}^{2}: C_{2} \leq \chi_{2(n+1)}^{2} \leq C_{1}\right\}
$$


From Chaturvedi and Malhotra (2016), the MLE and UMVUE of $R(t)$ are respectively given by:

$$
\hat{R}(t)=\exp \left\{\frac{-(n+1) t)}{R_{n}}\right\}
$$

and

$$
\tilde{R}(t)=\left\{\begin{array}{r}
{\left[1-\frac{t}{R_{n}}\right]^{n} ; t<R_{n} .} \\
0 ; \text { otherwise }
\end{array}\right.
$$

Thus, we define two different PTES of $R(t)$ based on MLE and UMVUE as follows:

and

$$
\begin{aligned}
& \hat{R}(t)^{P T}=\hat{R}(t)-\left(\hat{R}(t)-R_{o}(t)\right) I(A) \\
& \tilde{R}(t)^{P T}=\tilde{R}(t)-\left(\tilde{R}(t)-R_{o}(t)\right) I(A),
\end{aligned}
$$

where $R_{o}(t)=e^{\frac{-t}{\theta_{o}}}$

Let $X$ and $Y$ be two independent $r v s$ from exponential distribution with parameters $\theta_{1}$ and $\theta_{2}$ respectively. Let $R_{0}, R_{1}, \ldots, R_{n}$ be $n+1$ record values from distribution of $X$ and $R_{0}^{*}, R_{1}^{*}, \ldots, R_{m}^{*}$ be $m+1$ records from distribution of $Y$. Then, $P=\theta_{1}\left(\theta_{1}+\theta_{2}\right)^{-1}$. Suppose we want to test

$$
\begin{aligned}
& H_{\mathrm{o}}: P=P_{\mathrm{o}} \\
& H_{1}: P \neq P_{\mathrm{o}} .
\end{aligned}
$$

Note that $H_{\mathrm{o}}$ is equivalent to $\theta_{1}=k \theta_{2}$ where $k=P_{\mathrm{o}}\left(1-P_{\mathrm{o}}\right)^{-1}$. Thus, $H_{\mathrm{o}}: \theta_{1}=k \theta_{2}$ and $H_{1}: \theta_{1} \neq k \theta_{2}$. It follows from Chaturvedi and Malhotra (2016) that $\frac{(m+1) \theta_{2} R_{n}}{(n+1) \theta_{1} R_{m}^{*}} \sim F_{2(n+1), 2(m+1)}$ and the critical region is given by

$$
\left\{\frac{R_{n}}{R_{m}^{*}}<k_{2}\right\} \cup\left\{\frac{R_{n}}{R_{m}^{*}}>k_{2}^{\prime}\right\}
$$

where $k_{2}=\frac{k(n+1)}{(m+1)} F_{2(n+1), 2(m+1)}\left(\frac{\alpha}{2}\right)$ and $k_{2}{ }^{\prime}=\frac{k(n+1)}{(m+1)} F_{2(n+1), 2(m+1)}\left(1-\frac{\alpha}{2}\right)$. Thus, we define two PTES of $P$ based on MLE and UMVUE of $P$ as follows:

$$
\hat{P}^{P T}=\hat{P}-\left(\hat{P}-P_{o}\right) I(B)
$$

and

$$
\tilde{P}^{P T}=\tilde{P}-\left(\tilde{P}-P_{o}\right) I(B),
$$

where $I(B)$ is the indicator function of the set: 


$$
B=\left\{F_{2(n+1), 2(m+1)}: C_{4}<F_{2(n+1), 2(m+1)}<C_{3}\right\}
$$

Here, $C_{3}=F_{2(n+1), 2(m+1)}\left(1-\frac{\alpha}{2}\right), C_{4}=F_{2(n+1), 2(m+1)}\left(\frac{\alpha}{2}\right)$ and $\hat{P}$ and $\tilde{P}$ are the MLE and UMVUE of $P$ respectively as defined in Chaturvedi and Malhotra (2016) and are given by:

$$
\begin{gathered}
\hat{P}=\frac{(m+1) R_{n}}{(m+1) R_{n}+(n+1) R_{m}^{*}} \\
\tilde{P}=\left\{\begin{array}{ll}
\sum_{i=0}^{m-1} \frac{(-1)^{i} m ! n !}{(m-i-1) !(n+i+1) !}\left\{\frac{R_{n}}{R_{m}^{*}}\right\}^{i+1} ; R_{n}<R_{m}^{*} \\
\sum_{i=0}^{n} \frac{(-1)^{i} n ! m !}{(n-i) !(m+i) !}\left\{\frac{R_{m}^{*}}{R_{n}}\right\}^{i} ; R_{m}^{*}<R_{n}
\end{array} .\right.
\end{gathered}
$$

\section{Bias and Mean Square Error of the Preliminary test Estimators}

In this section, the following theorems provide the bias and MSE expressions for PTES based on UMVUE, MLE and EBE of $\theta^{p}, R(t)$ and $P$. We define $\lambda=\frac{\theta_{o}}{\theta}$.

Theorem 1: The bias and MSE of PTE of $\theta^{p}(p \in(-\infty, \infty), p \neq 0)$ based on UMVUE are

$$
\begin{aligned}
\operatorname{Bias}\left(\hat{\theta}_{U}^{p^{P T}}\right)= & \theta_{o}^{p}\left[\left\{H_{2 n+2}\left(\lambda C_{1}\right)-H_{2 n+2}\left(\lambda C_{2}\right)\right\}\right. \\
& \left.-\frac{1}{\lambda^{p}}\left\{H_{2(n+p+1)}\left(\lambda C_{1}\right)-H_{2(n+p+1)}\left(\lambda C_{2}\right)\right\}\right]
\end{aligned}
$$

and

$$
\begin{aligned}
\operatorname{MSE}\left(\hat{\theta}_{U}^{p^{P T}}\right)= & \theta^{2 p}\left[\frac{\Gamma(n+2 p+1) \Gamma(n+1)}{\Gamma^{2}(n+p+1)}-1\right] \\
& -\theta^{2 p} \frac{\Gamma(n+2 p+1) \Gamma(n+1)}{\Gamma^{2}(n+p+1)}\left\{H_{2(n+2 p+1)}\left(\lambda C_{1}\right)\right. \\
& \left.-H_{2(n+2 p+1)}\left(\lambda C_{2}\right)\right\} \\
& +\left(\theta_{o}^{2 p}-2 \theta^{p} \theta_{o}^{p}\right)\left\{H_{2 n+2}\left(\lambda C_{1}\right)-H_{2 n+2}\left(\lambda C_{2}\right)\right\} \\
& +2 \theta^{2 p}\left\{H_{2(n+p+1)}\left(\lambda C_{1}\right)-H_{2(n+p+1)}\left(\lambda C_{2}\right)\right\},
\end{aligned}
$$


where $H_{\gamma}($.$) stands for the c d f$ of $\chi^{2}$ distribution with $\gamma$ degrees of freedom.

Proof: It is easy to see that

$$
\operatorname{Bias}\left(\hat{\theta}_{U}^{p^{P T}}\right)=E\left[\hat{\theta}_{U}^{p}-\left(\hat{\theta}_{U}^{p}-\theta_{o}^{p}\right) I(A)-\theta^{p}\right] .
$$

Also, $\operatorname{MSE}\left(\hat{\theta}_{U}^{p P T}\right)=E\left[\left(\hat{\theta}_{U}^{p^{P T}}-\theta^{p}\right)^{2}\right]$

$$
=\operatorname{Var}\left(\hat{\theta}_{U}^{p^{P T}}\right)+\left[\operatorname{Bias}\left(\hat{\theta}_{U}^{p P T}\right)\right]^{2},
$$

where

$$
\begin{aligned}
\operatorname{Var}\left(\hat{\theta}_{U}^{p P T}\right)= & \operatorname{Var}\left(\hat{\theta}_{U}^{p}\right)+\operatorname{Var}\left(\left(\hat{\theta}_{U}^{p}-\theta_{o}^{p}\right) I(A)\right)-2 \operatorname{Cov}\left(\hat{\theta}_{U}^{p},\left(\hat{\theta}_{U}^{p}-\theta_{o}^{p}\right) I(A)\right) \\
= & \theta^{2 p}\left[\frac{\Gamma(n+2 p+1) \Gamma(n+1)}{\Gamma^{2}(n+p+1)}-1\right] \\
& +\theta^{2 p} \frac{\Gamma(n+2 p+1) \Gamma(n+1)}{\Gamma^{2}(n+p+1)}\left\{H_{2(n+2 p+1)}\left(\lambda C_{1}\right)-H_{2(n+2 p+1)}\left(\lambda C_{2}\right)\right\} \\
& -\theta^{2 p}\left\{H_{2(n+p+1)}\left(\lambda C_{1}\right)-H_{2(n+p+1)}\left(\lambda C_{2}\right)\right\}^{2} \\
& +\theta_{o}^{2 p}\left\{H_{2 n+2}\left(\lambda C_{1}\right)-H_{2 n+2}\left(\lambda C_{2}\right)\right\}\left[1-\left\{H_{2 n+2}\left(\lambda C_{1}\right)-H_{2 n+2}\left(\lambda C_{2}\right)\right\}\right] \\
& -2 \theta_{o}^{p} \theta^{p}\left\{H_{2(n+p+1)}\left(\lambda C_{1}\right)-H_{2(n+p+1)}\left(\lambda C_{2}\right)\right\}[1 \\
& \left.-\left\{H_{2 n+2}\left(\lambda C_{1}\right)-H_{2 n+2}\left(\lambda C_{2}\right)\right\}\right] \\
& -2 \theta^{2 p} \frac{\Gamma(n+2 p+1) \Gamma(n+1)}{\Gamma^{2}(n+p+1)}\left\{H_{2(n+2 p+1)}\left(\lambda C_{1}\right)-H_{2(n+2 p+1)}\left(\lambda C_{2}\right)\right\} \\
& +2 \theta_{o}^{p} \theta^{p}\left\{H_{2(n+p+1)}\left(\lambda C_{1}\right)-H_{2(n+p+1)}\left(\lambda C_{2}\right)\right\} \\
& +2 \theta^{2 p}\left\{H_{2(n+p+1)}\left(\lambda C_{1}\right)-H_{2(n+p+1)}\left(\lambda C_{2}\right)\right\} \\
& -2 \theta^{p} \theta_{o}^{p}\left\{H_{2 n+2}\left(\lambda C_{1}\right)-H_{2 n+2}\left(\lambda C_{2}\right)\right\}
\end{aligned}
$$

and the theorem follows.

Theorem 2: The bias and MSE of PTE of $\theta^{p}$ based on MLE are

$$
\begin{aligned}
\operatorname{Bias}\left(\hat{\theta}_{M L}^{p}{ }^{P T}\right) & =\left(\frac{\theta}{n+1}\right)^{p} \frac{\Gamma(n+p+1)}{\Gamma(n+1)}[1 \\
& \left.-\left\{H_{2(n+p+1)}\left(\lambda C_{1}\right)-H_{2(n+p+1)}\left(\lambda C_{2}\right)\right\}\right] \\
& +\theta_{o}^{p}\left\{H_{2 n+2}\left(\lambda C_{1}\right)-H_{2 n+2}\left(\lambda C_{2}\right)\right\}-\theta^{p}
\end{aligned}
$$

and 


$$
\begin{aligned}
M S E\left(\hat{\theta}_{M L}^{p}{ }^{P T}\right) & =\left\{\left(\frac{\theta}{n+1}\right)^{p} \frac{\Gamma(n+p+1)}{\Gamma(n+1)}\right\}^{2}\left[\frac{\Gamma(n+2 p+1) \Gamma(n+1)}{\Gamma^{2}(n+p+1)}-1\right] \\
& -\left(\frac{\theta}{n+1}\right)^{2 p} \frac{\Gamma(n+2 p+1)}{\Gamma(n+1)}\left\{H_{2(n+2 p+1)}\left(\lambda C_{1}\right)\right. \\
& \left.-H_{2(n+2 p+1)}\left(\lambda C_{2}\right)\right\} \\
& -\left\{\left(\frac{\theta}{n+1}\right)^{p} \frac{\Gamma(n+p+1)}{\Gamma(n+1)}\right\}^{2}\left\{H_{2(n+p+1)}\left(\lambda C_{1}\right)\right. \\
& \left.-H_{2(n+p+1)}\left(\lambda C_{2}\right)\right\}^{2} \\
& +\theta_{o}^{2 p}\left\{H_{2 n+2}\left(\lambda C_{1}\right)-H_{2 n+2}\left(\lambda C_{2}\right)\right\}[1 \\
& \left.-\left\{H_{2 n+2}\left(\lambda C_{1}\right)-H_{2 n+2}\left(\lambda C_{2}\right)\right\}\right] \\
& \left.+\theta_{o}^{p}\left\{H_{2 n+2}\left(\lambda C_{1}\right)-H_{2 n+2}\left(\lambda C_{2}\right)\right\}-\theta^{p}\right]^{2} \\
& +2 \theta_{o}^{p}\left(\frac{\theta}{n+1}\right)^{p} \frac{\Gamma(n+p+1)}{\Gamma(n+1)}\left[\left\{H_{2(n+p+1)}\left(\lambda C_{1}\right)\right.\right. \\
& \left.\left.-H_{2(n+p+1)}\left(\lambda C_{2}\right)\right\}-1\right] \\
& +2\left\{\left(\frac{\theta}{n+1}\right)^{p} \frac{\Gamma(n+p+1)}{\Gamma(n+1)}\right\}^{2}\left\{H_{2(n+p+1)}\left(\lambda C_{1}\right)\right. \\
& -\{(n+p+1) \\
\Gamma(n+1) & \left.\left(\lambda C_{2}\right)\right\} \\
& \\
& \\
&
\end{aligned}
$$

Theorem 3: The bias and MSE of PTE of $\theta^{p}$ based on EBE are

$$
\begin{aligned}
\operatorname{Bias}\left(\hat{\theta}_{E B}^{p}{ }^{P T}\right) & =\frac{\Gamma\left(n+\hat{v}_{M L}-p+1\right)}{\Gamma\left(n+\hat{v}_{M L}+1\right) \Gamma(n+1) \theta^{n+1}}\left(\varphi_{1}-\varphi_{3}\right) \\
& +\theta_{o}^{p}\left\{H_{2 n+2}\left(\lambda C_{1}\right)-H_{2 n+2}\left(\lambda C_{2}\right)\right\}-\theta^{p}
\end{aligned}
$$

and 


$$
\begin{aligned}
M S E\left(\hat{\theta}_{E B}^{p}{ }^{P T}\right) & =\left\{\frac{\Gamma\left(n+\hat{v}_{M L}-p+1\right)}{\Gamma\left(n+\hat{v}_{M L}+1\right)}\right\}^{2} \frac{\left(\varphi_{2}-\varphi_{4}\right)}{\Gamma(n+1) \theta^{n+1}} \\
& -\left[\frac{\Gamma\left(n+\hat{v}_{M L}-p+1\right)}{\Gamma\left(n+\hat{v}_{M L}+1\right) \Gamma(n+1) \theta^{n+1}}\right]^{2}\left(\varphi_{1}-\varphi_{3}\right)^{2} \\
& +\theta_{o}^{2 p}\left\{H_{2 n+2}\left(\lambda C_{1}\right)-H_{2 n+2}\left(\lambda C_{2}\right)\right\}[1 \\
& \left.-\left\{H_{2 n+2}\left(\lambda C_{1}\right)-H_{2 n+2}\left(\lambda C_{2}\right)\right\}\right] \\
& +2 \theta_{o}^{p} \frac{\Gamma\left(n+\hat{v}_{M L}-p+1\right)}{\Gamma\left(n+\hat{v}_{M L}+1\right) \Gamma(n+1) \theta^{n+1}}\left(\varphi_{3}-\varphi_{1}\right)\left\{H_{2 n+2}\left(\lambda C_{1}\right)\right. \\
& \left.-H_{2 n+2}\left(\lambda C_{2}\right)\right\} \\
& +\left[\frac{\Gamma\left(n+\hat{v}_{M L}-p+1\right)}{\Gamma\left(n+\hat{v}_{M L}+1\right) \Gamma(n+1) \theta^{n+1}}\left(\varphi_{1}-\varphi_{3}\right)\right. \\
& \left.+\theta_{o}^{p}\left\{H_{2 n+2}\left(\lambda C_{1}\right)-H_{2 n+2}\left(\lambda C_{2}\right)\right\}-\theta^{p}\right]^{2},
\end{aligned}
$$

where $\varphi_{1}=\int_{0}^{\infty}\left(y+\hat{\mu}_{M L}\right)^{p} y^{n} e^{\frac{-y}{\theta}} d y, \varphi_{2}=\int_{0}^{\infty}\left(y+\hat{\mu}_{M L}\right)^{2 p} y^{n} e^{\frac{-y}{\theta}} d y$, $\varphi_{3}=\int_{\frac{\theta_{o} C_{2}}{2}}^{\frac{\theta_{o} C_{1}}{2}}\left(y+\hat{\mu}_{M L}\right)^{p} y^{n} e^{\frac{-y}{\theta}} d y$ and $\varphi_{4}=\int_{\frac{\theta_{o} C_{2}}{2}}^{\frac{\theta_{o} C_{1}}{2}}\left(y+\hat{\mu}_{M L}\right)^{2 p} y^{n} e^{\frac{-y}{\theta}} d y$.

The results of Theorem 2 and 3 can derived on the same lines as the proof of Theorem 1.

Theorem 4: The bias and MSE of PTES of $R(t)$ based on MLE are

$$
\begin{aligned}
\operatorname{Bias}\left(\hat{R}(t)^{P T}\right) & =\frac{2}{n !}\left\{\frac{(n+1) t}{\theta}\right\}^{\frac{n+1}{2}} K_{n+1}\left(2 \sqrt{\frac{(n+1) t}{\theta}}\right)-\varphi_{5} \\
& +R_{o}(t)\left\{H_{2 n+2}\left(\lambda C_{1}\right)-H_{2 n+2}\left(\lambda C_{2}\right)\right\}-R(t)
\end{aligned}
$$

and 


$$
\begin{aligned}
M S E\left(\hat{R}(t)^{P T}\right)= & \frac{2}{n !}\left\{\frac{2(n+1) t}{\theta}\right\}^{\frac{n+1}{2}} K_{n+1}\left(2 \sqrt{\frac{2(n+1) t}{\theta}}\right) \\
& -\left[\frac{2}{n !}\left\{\frac{(n+1) t}{\theta}\right\}^{\frac{n+1}{2}} K_{n+1}\left(2 \sqrt{\left.\frac{(n+1) t}{\theta}\right)}\right]^{2}+\varphi_{6}-\varphi_{5}{ }^{2}\right. \\
& +R_{o}(t)\left\{H_{2 n+2}\left(\lambda C_{1}\right)-H_{2 n+2}\left(\lambda C_{2}\right)\right\}\{1 \\
& \left.-\left\{H_{2 n+2}\left(\lambda C_{1}\right)-H_{2 n+2}\left(\lambda C_{2}\right)\right\}\right\} \\
& +2\left[\frac{2}{n !}\left\{\frac{(n+1) t}{\theta}\right\}^{\frac{n+1}{2}} K_{n+1}\left(2 \sqrt{\frac{(n+1) t}{\theta}}\right)\right] \varphi_{5} \\
& +2 R_{o}(t)\left\{H_{2 n+2}\left(\lambda C_{1}\right)\right. \\
& \left.\left.-H_{2 n+2}\left(\lambda C_{2}\right)\right\}\left(\frac{2}{n !}\left\{\frac{(n+1) t}{\theta}\right\}\right]^{\frac{n+1}{2}}\left(2 \sqrt{\frac{(n+1) t}{\theta}}\right)-\varphi_{5}\right) \\
& +\left[\frac{2}{n !}\left\{\frac{(n+1) t}{\theta}\right\}^{\frac{n+1}{2}} K_{n+1}(t)\left\{H_{2 n+2}\left(\lambda C_{1}\right)-H_{2 n+2}\left(\lambda C_{2}\right)\right\}-R(t)\right. \\
& {\left[\frac{(n+1) t}{\theta}\right)-\varphi_{5} }
\end{aligned}
$$

where

$$
\varphi_{5}=\int_{C_{2} / 2}^{C_{1} / 2} \frac{z^{n}}{n !} \exp \left(-\left(z+\frac{(n+1) t}{\theta z}\right)\right) d z \text { and } \varphi_{6}=\int_{C_{2} / 2}^{C_{1} / 2} \frac{z^{n}}{n !} \exp \left(-\left(z+\frac{2(n+1) t}{\theta z}\right)\right) d z .
$$

Proof: We can write

$$
\begin{aligned}
\operatorname{Bias}\left(\hat{R}(t)^{P T}\right) & =\frac{1}{\Gamma(n+1)} \int_{0}^{\infty} \exp \left[-\left\{y+\frac{(n+1) t}{\theta y}\right\}\right] y^{n} d y-\varphi_{5} \\
& +R_{o}(t)\left\{H_{2 n+2}\left(\lambda C_{1}\right)-H_{2 n+2}\left(\lambda C_{2}\right)\right\}-R(t)
\end{aligned}
$$

Applying a result of Watson (1952),

$$
\int_{0}^{\infty} u^{-m} \exp \left\{-\left(a u+\frac{b}{u}\right)\right\} d u=2\left(\frac{a}{b}\right)^{\frac{m-1}{2}} K_{m-1}(2 \sqrt{a b})
$$

[it is to be noted that $K_{-m}(\cdot)=K_{m}(\cdot)$ for $m=0,1,2, \ldots$ ], and the theorem follows.

Theorem 5: The bias and MSE of PTE of $R(t)$ based on UMVUE are 


$$
\operatorname{Bias}\left(\tilde{R}(t)^{P T}\right)=R_{o}(t)\left\{H_{2 n+2}\left(\lambda C_{1}\right)-H_{2 n+2}\left(\lambda C_{2}\right)\right\}-\varphi_{8}
$$

and

$$
\begin{aligned}
\operatorname{MSE}\left(\tilde{R}(t)^{P T}\right) & =R-\varphi_{7}-\varphi_{8}{ }^{2} \\
& +R_{o}(t)\left\{H_{2 n+2}\left(\lambda C_{1}\right)-H_{2 n+2}\left(\lambda C_{2}\right)\right\}\{1 \\
& \left.-\left\{H_{2 n+2}\left(\lambda C_{1}\right)-H_{2 n+2}\left(\lambda C_{2}\right)\right\}\right\}+2 \varphi_{8} R(t) \\
& +2 R_{o}(t)\left\{H_{2 n+2}\left(\lambda C_{1}\right)-H_{2 n+2}\left(\lambda C_{2}\right)\right\}\left(\varphi_{8}-R(t)\right) \\
& +\left[R_{o}(t)\left\{H_{2 n+2}\left(\lambda C_{1}\right)-H_{2 n+2}\left(\lambda C_{2}\right)\right\}-\varphi_{8}\right]^{2},
\end{aligned}
$$

where

$$
\varphi_{7}=\int_{C_{2}}^{C_{1}}\left(1-\frac{2 t}{\theta u}\right)^{2 n} \frac{u^{n} e^{\frac{-u}{2}}}{2^{n+1} n !} d u \text { and } \varphi_{8}=\int_{C_{2}}^{C_{1}}\left(1-\frac{2 t}{\theta u}\right)^{n} \frac{u^{n} e^{\frac{-u}{2}}}{2^{n+1} n !} d u .
$$

Proof: From Chaturvedi and Malhotra (2016),

$$
\begin{aligned}
\operatorname{Var}\{\tilde{R}(t)\}= & \frac{1}{n !}\left\{\frac{t}{\theta}\right\}^{(n+1)} \exp \left\{\frac{-t}{\theta}\right\}\left[\frac{\theta a_{n}}{t}-a_{n-1} \exp \left\{\frac{t}{\theta}\right\} E_{i}\left(-\frac{t}{\theta}\right)\right. \\
& +\sum_{i=0}^{n-2} a_{i}\left\{\sum_{m=1}^{n-i-1} \frac{(m-1) !}{(n-i-1) !}\left(-\frac{t}{\theta}\right)^{n-i-m-1}\right. \\
& \left.-\frac{1}{(n-i-1) !}\left(-\frac{t}{\theta}\right)^{n-i-1} \exp \left(\frac{t}{\theta}\right) E_{i}\left(-\frac{t}{\theta}\right)\right\} \\
& \left.+\sum_{i=n+1}^{2 n} a_{i}(i-n) !\left(\frac{\theta}{t}\right)^{i-n+1} \sum_{r=0}^{i-n} \frac{1}{r !}\left(\frac{t}{\theta}\right)^{r}\right]-\exp \left\{\frac{-2 t}{\theta}\right\} \\
= & R(\text { say }),
\end{aligned}
$$

where $a_{i}=(-1)^{i}\left(\begin{array}{c}2 n \\ i\end{array}\right)$ and $-E_{i}(-x)=\int_{x}^{\infty} \frac{e^{-u}}{u} d u$. The results can now be easily derived.

Theorem 6: The bias and MSE of PTE of $P$ based on MLE are

$$
\begin{aligned}
& \operatorname{Bias}\left(\hat{P}^{P T}\right) \\
& =\left\{\begin{array}{r}
-\varphi_{10}+P_{o}\left\{F_{2(n+1), 2(m+1)}\left(C_{3}\right)-F_{2(n+1), 2(m+1)}\left(C_{4}\right)\right\} ; \omega=1 \\
\varphi_{9}-\varphi_{10}+P_{o}\left\{F_{2(n+1), 2(m+1)}\left(C_{3}\right)-F_{2(n+1), 2(m+1)}\left(C_{4}\right)\right\}-P ; \omega \neq 1
\end{array}\right.
\end{aligned}
$$

and 


$$
\begin{aligned}
& \operatorname{MSE}\left(\hat{P}^{P T}\right) \\
& =\left\{\begin{array}{c}
\left(\frac{n+1}{n+m+2}\right)^{2}\left[\left(\frac{n+2}{n+1}\right)\left(\frac{n+m+2}{n+m+3}\right)-1\right]-\varphi_{11}+2 P\left(\varphi_{10}-P_{o} P(B)\right)+P_{o}^{2} P(B) ; \omega=1 \\
\varphi_{12}-\varphi_{11}+2 P\left(\varphi_{10}-P_{o} P(B)\right)+P_{o}^{2} P(B)+P\left(P-2\left(\frac{n+1}{n+m+2}\right)\right) ; \omega \neq 1
\end{array},\right.
\end{aligned}
$$

where

$$
\begin{gathered}
\varphi_{9}=\frac{(-1)^{n+1} \omega^{-1}\left(1-\omega^{-1}\right)^{-n-m-2}}{\beta(n+1, m+1)} \sum_{j=0}^{m}(-1)^{j}\left(\begin{array}{c}
m \\
j
\end{array}\right) \omega^{-j} \sum_{k=0}^{n+1}(-1)^{k}\left(\begin{array}{c}
n+1 \\
k
\end{array}\right) I(\omega ; j+k-n-m-2), \\
\varphi_{10}=\frac{1}{\beta(n+1, m+1)}\left(\frac{n+1}{m+1}\right)^{n+1} \int_{C_{4}}^{C_{3}} \frac{u^{n}\left(1+\left(\frac{n+1}{m+1}\right) u\right)^{-n-m-2}}{\left(1+\frac{\theta_{2}}{\theta_{1} u}\right)} d u, \\
\varphi_{11}=\frac{1}{\beta(n+1, m+1)}\left(\frac{n+1}{m+1}\right)^{n+1} \int_{C_{4}}^{C_{3}} \frac{u^{n}\left(1+\left(\frac{n+1}{m+1}\right) u\right)^{-n-m-2}}{\left(1+\frac{\theta_{2}}{\theta_{1} u}\right)^{2}} d u
\end{gathered}
$$

and

$$
\varphi_{12}=\frac{(-1)^{n} \omega^{-2}\left(1-\omega^{-1}\right)^{-n-m-3}}{\beta(n+1, m+1)} \sum_{j=0}^{m}(-1)^{j}\left(\begin{array}{c}
m \\
j
\end{array}\right) \omega^{-j} \sum_{k=0}^{n+2}(-1)^{k}\left(\begin{array}{c}
n+2 \\
k
\end{array}\right) I(\omega ; j+k-n-m-2) .
$$

Proof: We can write

$$
\operatorname{Bias}\left(\hat{P}^{P T}\right)=E(\widehat{P})-E(\widehat{P} I(B))+P_{o} E(I(B))-P
$$

and

$$
\begin{aligned}
E(\hat{P}) & =E\left(\frac{\widehat{\theta_{1}}}{\widehat{\theta_{1}}+\widehat{\theta_{2}}}\right) \\
& =E(\widehat{Q}),(\text { say })
\end{aligned}
$$

Following the approach by Constantine et al. (1986), we obtain the $p d f$ of $\hat{Q}$ by transformation into two new independent $r v s r>0$ and $\theta \in\left(0, \frac{\pi}{2}\right)$ where $\widehat{\theta_{1}}=\frac{\theta_{1} r \sin ^{2} \theta}{n+1}$ and $\widehat{\theta_{2}}=\frac{\theta_{2} r \cos ^{2} \theta}{m+1}$. Putting $\varphi=\cos ^{2} \theta$, the $p d f$ of $\hat{Q}=\left[1+\rho\left(\frac{m+1}{n+1}\right)\left(\frac{\varphi}{1-\varphi}\right)\right]^{-1}$ is

$$
\begin{gathered}
g(q)=\frac{1}{\beta(n+1, m+1)}\left(\rho\left(\frac{m+1}{n+1}\right)\right)^{n+1} \frac{q^{n}(1-q)^{m}}{(1+\varepsilon q)^{n+m+2}} \\
0<q<1, \varepsilon=\rho\left(\frac{m+1}{n+1}\right)-1 .
\end{gathered}
$$

When $\varepsilon=0$, equation (37) gives: 


$$
E\left(\hat{Q}^{l}\right)=\frac{\beta(n+l+1, m+1)}{\beta(n+1, m+1)}
$$

When $\varepsilon \neq 0$, equation (37) yields on substituting $1+\varepsilon q=t$,

$$
E\left(\hat{Q}^{l}\right)=\frac{1}{\beta(n+1, m+1)}\left(\rho\left(\frac{m+1}{n+1}\right)\right)^{n+1} \frac{1}{\varepsilon^{n+m+l+1}} \int_{1}^{\omega}(t-1)^{n+l}(c-t)^{m} t^{-(n+m+2)} d t
$$

where $\omega=1+\varepsilon$. Two binomial expansions further simplify equation (39) to

$$
\begin{aligned}
E\left(\hat{Q}^{l}\right)= & \frac{(-1)^{n+l} \omega^{-l}\left(1-\omega^{-1}\right)^{-n-m-l-1}}{\beta(n+1, m+1)} \sum_{j=0}^{m}(-1)^{j}\left(\begin{array}{c}
m \\
j
\end{array}\right) \omega^{-j} \\
& \sum_{k=0}^{n+l}(-1)^{k}\left(\begin{array}{c}
n+l \\
k
\end{array}\right) I(\omega ; j+k-n-m-2),
\end{aligned}
$$

where $I(\omega ; z)=\left\{\begin{array}{c}\frac{\omega^{z+1}-1}{z+1} ; z \neq-1 \\ \log (\omega) ; z=-1\end{array}\right.$ Thus,

$$
E(\hat{P})=\left\{\begin{array}{c}
\frac{n+1}{n+m+2} ; \omega=1 \\
\varphi_{9} ; \omega \neq 1
\end{array}\right.
$$

Now,

$$
\begin{aligned}
E(\hat{P} I(B)) & =\frac{1}{\beta(n+1, m+1)}\left(\frac{n+1}{m+1}\right)^{n+1} \int_{C_{4}}^{C_{3}} \frac{u^{n}\left(1+\left(\frac{n+1}{m+1}\right) u\right)^{-n-m-2}}{\left(1+\frac{\theta_{2}}{\theta_{1} u}\right)} d u \\
& =\varphi_{10}, \text { (say). }
\end{aligned}
$$

Then the bias and MSE of PTE of $P$ based on MLE can be derived.

Theorem 7: The bias and MSE of PTE of $P$ based on UMVUE are

$$
\operatorname{Bias}\left(\tilde{P}^{P T}\right)=\left\{\begin{array}{l}
P_{o} P(B)-\varphi_{13} ; v \leq 1 \\
P_{o} P(B)-\varphi_{14} ; v>1
\end{array}\right.
$$

and

$$
\operatorname{MSE}\left(\tilde{P}^{P T}\right)=\left\{\begin{array}{l}
\varphi_{17}-P^{2}-\varphi_{15}+2 P\left(\varphi_{13}-P_{o} P(B)\right)+P_{o}^{2} P(B) ; v \leq 1 \\
\varphi_{17}-P^{2}-\varphi_{16}+2 P\left(\varphi_{14}-P_{o} P(B)\right)+P_{o}^{2} P(B) ; v>1
\end{array},\right.
$$


where $v=\frac{R_{n}}{R_{m}^{*}}, \varphi_{13}=\sum_{i=0}^{m-1} \frac{(-1)^{i} m ! n !}{(m-i-1) !(n+i+1) !}\left(\frac{\theta_{1}(n+1)}{\theta_{2}(m+1)}\right)^{i+1} \int_{C_{4}}^{C_{3}} u^{i+1} \emptyset_{1}(u) d u$, where $\emptyset_{1}(\cdot)$ is the $p d f$ of $f-$ distribution with $(2(n+1), 2(m+1))$ degrees of freedom,

$\varphi_{14}=\sum_{i=0}^{n} \frac{(-1)^{i} n ! m !}{(n-i) !(m+i) !}\left(\frac{\theta_{2}(m+1)}{\theta_{1}(n+1)}\right)^{i} \int_{C_{4}}^{C_{3}} u^{i} \emptyset_{2}(u) d u$, where $\emptyset_{2}(\cdot)$ is the $p d f$ of $f$-distribution with $(2(m+1), 2(n+1))$ degrees of freedom,

$$
\begin{aligned}
\varphi_{15} & =\sum_{i=0}^{m-1} \sum_{j=0}^{m-1} a_{i} a_{j}\left(\frac{\theta_{1}(n+1)}{\theta_{2}(m+1)}\right)^{i+j+2} \int_{C_{4}}^{C_{3}} u^{i+j+2} \emptyset_{1}(u) d u, \\
\varphi_{16} & =\sum_{i=0}^{n} \sum_{j=0}^{n} b_{i} b_{j}\left(\frac{\theta_{2}(m+1)}{\theta_{1}(n+1)}\right)^{i+j} \int_{C_{4}}^{C_{3}} u^{i+j} \emptyset_{2}(u) d u \text { and } \\
\varphi_{17}= & \sum_{i=0}^{m-1} \sum_{j=0}^{m-1} \frac{a_{i} a_{j} \rho^{-(i+j+2)}}{\beta(n+1, m+1)} \sum_{k=0}^{n+i+j+2}(-1)^{k}\left(\begin{array}{c}
n+i+j+2 \\
k
\end{array}\right) \int_{Q}^{1} r^{m+k-i-j-2} d r \\
& +\sum_{i=0}^{n} \sum_{j=0}^{n} \frac{b_{i} b_{j} \rho^{i+j}}{\beta(n+1, m+1)} \sum_{k=0}^{m+i+j}(-1)^{k}\left(\begin{array}{c}
m+i+j \\
k
\end{array}\right) \int_{1-Q}^{1} r^{n+k-i-j} d r .
\end{aligned}
$$

Proof: To obtain $\operatorname{MSE}\left(\widetilde{P}^{P T}\right)$, consider

$$
E\left(\tilde{P}^{2}\right)=E\left(\sum_{i=0}^{m-1} \sum_{j=0}^{m-1} a_{i} a_{j}(v)^{i+j+2} \mid v \leq 1\right) P(v \leq 1)+E\left(\sum_{i=0}^{n} \sum_{j=0}^{n} b_{i} b_{j}(v)^{-(i+j)} \mid v>1\right) P(v>1),
$$

where, $a_{i}=\frac{(-1)^{i} m ! n !}{(m-i-1) !(n+i+1) !}$ and $b_{i}=\frac{(-1)^{i} n ! m !}{(n-i) !(m+i) !}$. An explicit expression of $\operatorname{Var}(\tilde{P})$ depends on the evaluation of $E\left(v^{l} \mid v \leq 1\right) P(v \leq 1)$ and $E\left(v^{-l} \mid v>1\right) P(v>1)$ for $l \geq 0$. To evaluate them we first obtain the $p d f$ of $v$. We have, $v=\frac{R_{n}}{R_{m}^{*}}$ which implies, $\frac{(m+1) \theta_{2}}{(n+1) \theta_{1}} v=$ $\rho \frac{(m+1)}{(n+1)} v \sim F_{2(n+1), 2(m+1)}$. Thus we obtain the $p d f$ of $v$ as:

$$
h(v)=\frac{\rho^{n-1}}{\beta(n+1, m+1)} v^{n}(1+\rho v)^{-n-m-2} ; v>0 .
$$

For $l>0$,

$$
E\left(v^{l} \mid v \leq 1\right) P(v \leq 1)=\int_{0}^{1} \frac{\rho^{n-1}}{\beta(n+1, m+1)} v^{n+l}(1+\rho v)^{-n-m-2} d v .
$$

Substituting $r=(1+\rho v)^{-1}$, the binomial expansion of the integrand yields,

$$
E\left(v^{l} \mid v \leq 1\right) P(v \leq 1)=\frac{\rho^{-l}}{\beta(n+1, m+1)} \sum_{k=0}^{n+l}(-1)^{k}\left(\begin{array}{c}
n+l \\
k
\end{array}\right) \int_{Q}^{1} r^{m-l+k} d r
$$

where 


$$
\int_{Q}^{1} r^{m-l+k} d r=\left\{\begin{array}{c}
\frac{1-Q^{m-l+k+1}}{m-l+k+1} ; k \neq l-m-1 \\
-\log (Q) ; k=l-m-1
\end{array}\right.
$$

and $Q=\frac{1}{1+\rho}$. Similarly we can obtain,

$$
E\left(v^{-l} \mid v>1\right) P(v>1)=\frac{\rho^{l}}{\beta(n+1, m+1)} \sum_{k=0}^{m+l}(-1)^{k}\left(\begin{array}{c}
m+l \\
k
\end{array}\right) \int_{1-Q}^{1} r^{n-l+k} d r
$$

where

$$
\int_{1-Q}^{1} r^{n-l+k} d r=\left\{\begin{array}{c}
\frac{1-(1-Q)^{n-l+k+1}}{n-l+k+1} ; k \neq l-n-1 \\
-\log (1-Q) ; k=l-n-1
\end{array}\right.
$$

Thus, $\operatorname{Var}(\tilde{P})=\varphi_{17}-P^{2}$ and $\operatorname{Var}(\tilde{P} I(B))=\left\{\begin{array}{l}\varphi_{15}-\varphi_{13}^{2} ; v \leq 1 \\ \varphi_{16}-\varphi_{14}^{2} ; v>1\end{array}\right.$ and we can obtain the MSE of PTE of $P$ based on UMVUE.

\section{Numerical Findings}

In this section, we study the relative efficiency of the proposed PTES over the existing estimators of our parameters based on MLE, UMVUE and EBE through simulation studies. Suppose we want to test the hypothesis $H_{o}: \theta=2$ against $H_{1}: \theta \neq 2$. Then using equations (26) and (28) we compute the MSE of $\hat{\theta}_{U}^{p^{P T}}$ and $\hat{\theta}_{M L}^{p{ }^{P T}}$ respectively. Since the relative efficiency of $\hat{\theta}_{M L}^{p}{ }^{P T}$ over $\hat{\theta}_{M L}^{p}$ and the relative efficiency of $\hat{\theta}_{U}^{p^{P T}}$ over $\hat{\theta}_{U}^{p}$ depend on the sample size $(n+1)$ and the level of significance $\alpha$, Table 1 and Figure 1show the relative efficiency of $\hat{\theta}_{M L}^{p P T}$ over $\hat{\theta}_{M L}^{p}$ where we take the power of $\theta, p=1$. Similarly, Table 2 and Figure 2 show the relative efficiency of $\hat{\theta}_{U}^{p P T}$ over $\hat{\theta}_{U}^{p}$ for $p=1$. Since the relative efficiency of $\hat{\theta}_{E B}^{p}{ }^{P T}$ over $\hat{\theta}_{E B}^{p}$ does not have a closed form and thus we use Monte Carlo simulation technique that involves the following steps:

a) For given values of $\mu$ and $v$, generate one sample from inverted-gamma $(\mu, v)$ and denote it as $\theta^{*}$.

b) For a specified value of $n$, generate $m$ random $\operatorname{samples}$ from $\operatorname{gamma}\left(n+1, \theta^{*}\right)$ to obtain $Y_{j} ; j=1,2, \ldots, m$.

c) Compute, $\hat{\theta}_{E B}^{p}(j)=\frac{\Gamma(n+v-p+1)}{\Gamma(n+v+1)}\left(Y_{j}+\mu\right)^{p} ; j=1,2, \ldots, m$. 
d) For a specified value of $\theta_{o}$, test the hypothesis $H_{o}: \theta=\theta_{o}$, using the test statistic in equation (13) to get $\hat{\theta}_{E B}^{p}{ }^{P T}(j)=\hat{\theta}_{E B}^{p}(j)-\left(\hat{\theta}_{E B}^{p}(j)-\theta_{o}^{p}\right) I(A) ; j=1,2, \ldots, m$.

e) Compute $\operatorname{MSE}=\frac{1}{m} \sum_{j=1}^{m}\left(\delta(j)-\theta^{* p}\right)^{2}$, where $\delta(j) \in\left\{\hat{\theta}_{E B}^{p}(j), \hat{\theta}_{E B}^{p}{ }^{P T}(j)\right\}, \quad j=$ $1,2, \ldots, m$.

For $\mu=2, v=4$ and $m=1000$, Table 3 and Figure 3 show the relative efficiency of $\hat{\theta}_{E B}^{p}{ }^{P T}$ over $\hat{\theta}_{E B}^{p}$ for $p=1$. From Tables 1,2 and 3, we observe that irrespective of the sample size and level of significance, the PTES based on MLE, UMVUE and EBE are always more efficient. Figures 1,2 and 3 also show that the PTES of $\theta^{p}$ perform better than the usual estimators of $\theta^{p}$ when the true parameter is close to the hypothesised value. Also we note that for larger sample sizes, PTES based on MLE and UMVUE become more efficient. However, for large samples, PTE of $\theta^{p}$ based on EBE tends to be as efficient as the EBE of $\theta^{p}$.

Table 1: Relative efficiency of PTE of $\theta^{p}$ based on MLE over MLE of $\theta^{p}$ for various sample sizes $n$ and level of significance $\alpha$.

\begin{tabular}{|c|c|c|c|c|c|}
\hline$n$ & 0.01 & 0.05 & 0.10 & 0.15 & 0.20 \\
\hline 5 & 60.8891 & 17.9925 & 11.0401 & 8.4115 & 6.9856 \\
\hline 10 & 128.8689 & 36.7107 & 22.0769 & 16.5914 & 13.6295 \\
\hline 15 & 204.7047 & 57.3397 & 34.1665 & 25.5167 & 20.8579 \\
\hline 20 & 284.4596 & 78.8995 & 46.7617 & 34.7966 & 28.3619 \\
\hline 30 & 449.7725 & 123.3742 & 72.6814 & 53.8643 & 43.7631 \\
\hline 60 & 961.9811 & 260.5351 & 152.4339 & 112.4477 & 91.0294 \\
\hline 80 & 1307.8877 & 352.9803 & 206.1340 & 151.8698 & 122.8214 \\
\hline
\end{tabular}

Table 2: Relative efficiency of PTE of $\theta^{p}$ based on UMVUE over UMVUE of $\theta^{p}$ for various sample sizes $n$ and level of significance $\alpha$.

\begin{tabular}{|c|c|c|c|c|c|}
\hline$n^{\alpha}$ & 0.01 & 0.05 & 0.10 & 0.15 & 0.20 \\
\hline 5 & 20.9229 & 6.2602 & 3.8672 & 2.9599 & 2.4670 \\
\hline 10 & 25.4327 & 7.2944 & 4.4030 & 3.3173 & 2.7306 \\
\hline 15 & 27.7466 & 7.8063 & 4.6627 & 3.4880 & 2.8549 \\
\hline 20 & 29.1546 & 8.1118 & 4.8160 & 3.5880 & 2.9272 \\
\hline 30 & 30.7819 & 8.4595 & 4.9889 & 3.7000 & 3.0079 \\
\hline 60 & 32.6841 & 8.8587 & 5.1854 & 3.8264 & 3.0983 \\
\hline 80 & 33.2109 & 8.9680 & 5.2388 & 3.8605 & 3.1227 \\
\hline
\end{tabular}


Table 3: Relative efficiency of PTE of $\theta^{p}$ based on EBE over EBE of $\theta^{p}$ for various sample sizes $n$ and level of significance $\alpha$.

\begin{tabular}{|c|c|c|c|c|c|}
\hline$n^{\alpha}$ & 0.01 & 0.05 & 0.10 & 0.15 & 0.20 \\
\hline 5 & 71.7660 & 71.7660 & 71.7660 & 71.7660 & 2.9114 \\
\hline 10 & 43.8590 & 43.8590 & 43.8590 & 43.8590 & 2.4381 \\
\hline 15 & 30.7385 & 30.7385 & 30.7385 & 30.7385 & 2.2091 \\
\hline 20 & 23.3446 & 23.3446 & 23.3446 & 23.3446 & 2.0686 \\
\hline 30 & 15.4818 & 15.4818 & 15.4818 & 15.4818 & 1.8948 \\
\hline 60 & 7.4154 & 7.4154 & 7.4154 & 1.6268 & 1.6268 \\
\hline 80 & 5.4366 & 5.4366 & 5.4366 & 1.5123 & 1.1774 \\
\hline
\end{tabular}

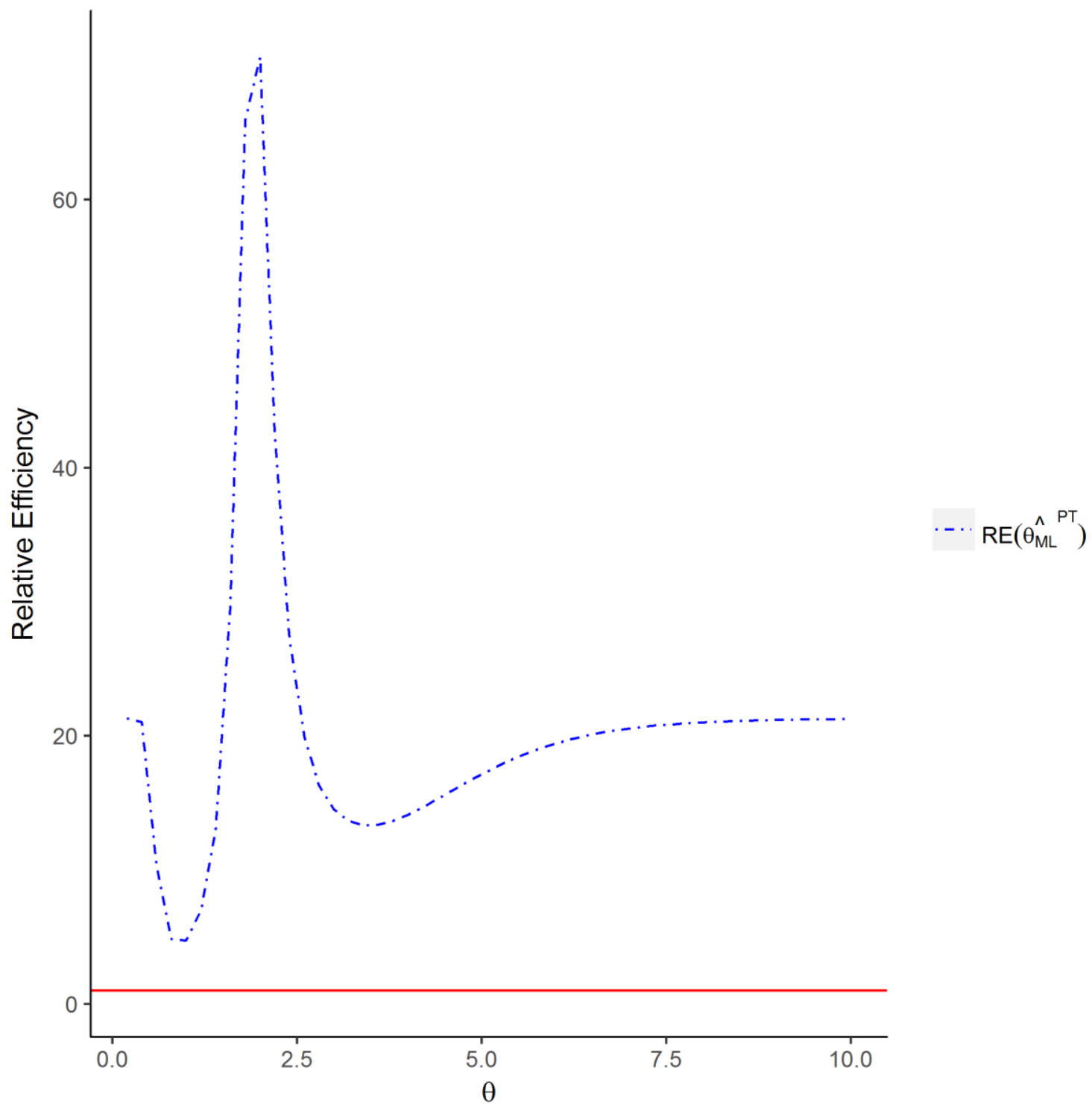

Figure 1: Relative Efficiency of $\hat{\theta}_{M L}{ }^{P T}$ over $\widehat{\theta}_{M L}$. 


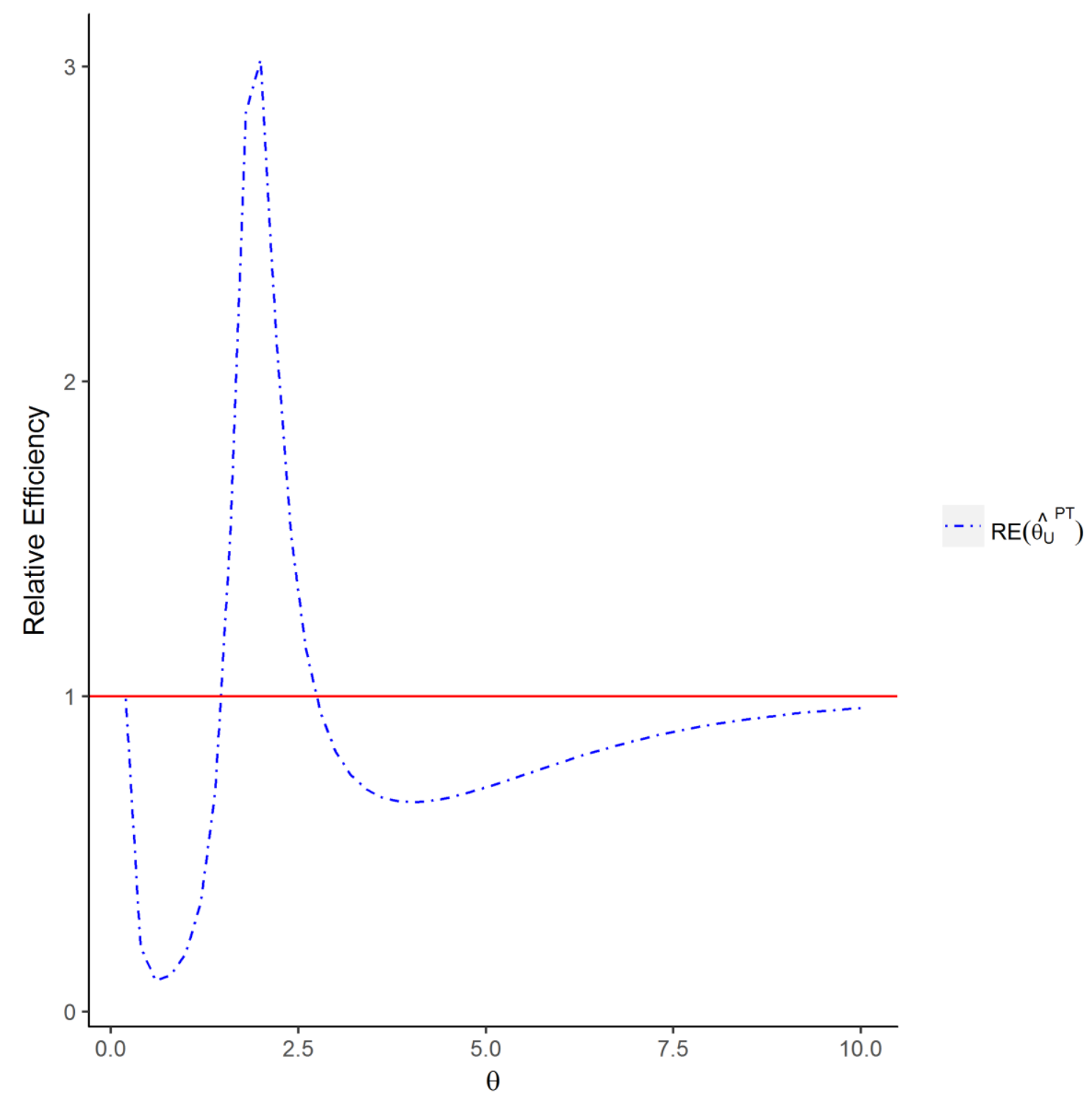

Figure 2: Relative Efficiency of $\hat{\theta}_{U}{ }^{P T}$ over $\widehat{\theta}_{U}$ 


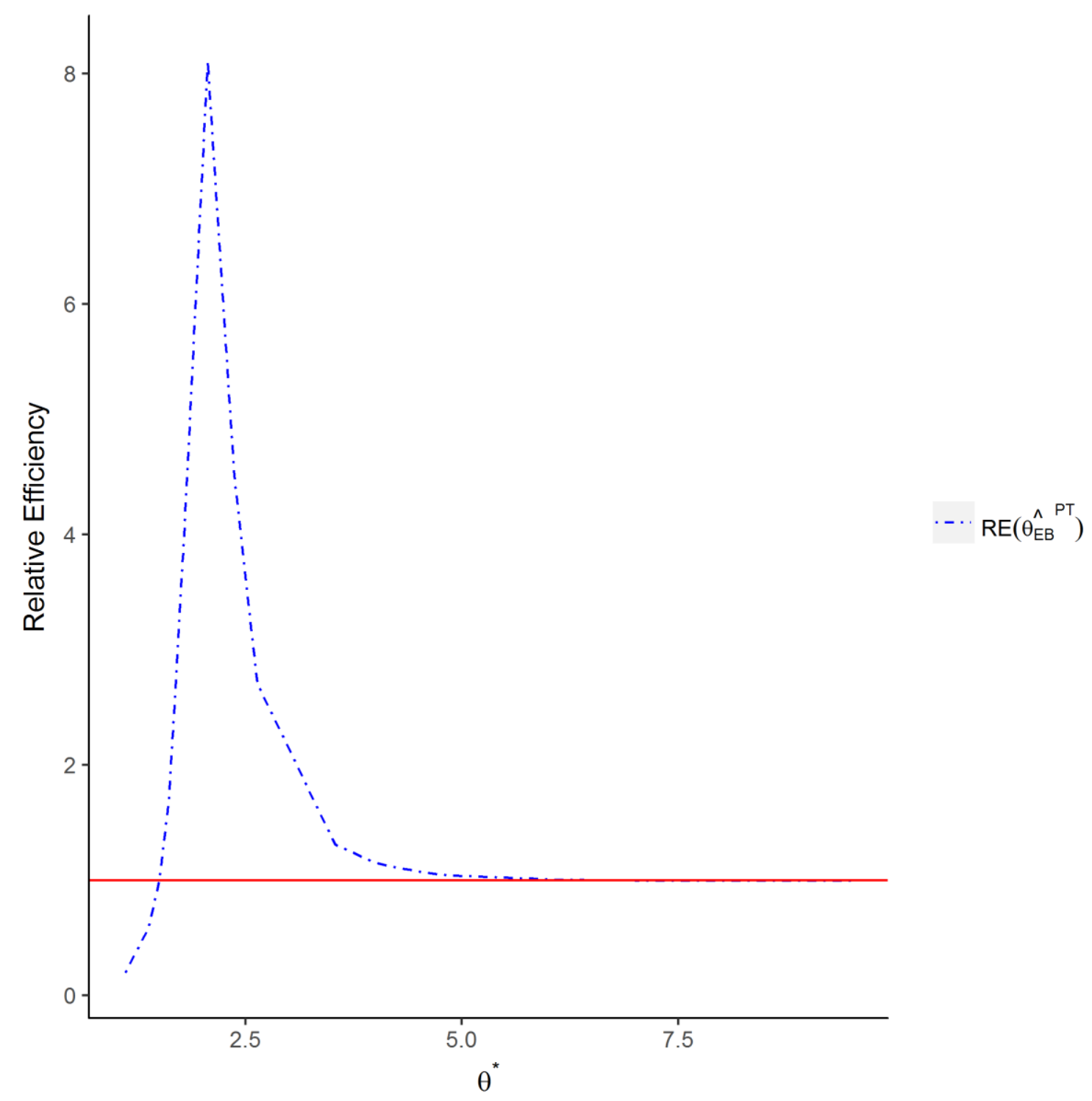

Figure 3: Relative Efficiency of $\hat{\theta}_{E B}{ }^{P T}$ over $\hat{\theta}_{E B}$

Using the same technique as used for obtaining results in Table 3 , for a fixed sample size, $n=20$, Table 4 and 5 show the relative efficiency of $\hat{R}(t)^{P T}$ over $\hat{R}(t)$ and $\tilde{R}(t)^{P T}$ over $\tilde{R}(t)$ respectively for different time points and level of significance. Figures 4 and 5 illustrate the relative efficiency of PTE over the usual estimator of $R(t)$ based on MLE and UMVUE respectively. From these Tables and Figures, it is clear that PTES of $R(t)$ based on MLE and UMVUE outperform the usual estimators of $R(t)$ whenever the true parameter is close to the hypothesised value.

Table 4: Relative efficiency of PTE of $R(t)$ based on MLE over MLE of $R(t)$ for different time points $t$ and level of significance $\alpha$. 


\begin{tabular}{|c|c|c|c|c|c|c|}
\hline$t$ & $\begin{array}{c}\alpha(t)^{\alpha}\end{array}$ & 0.01 & 0.05 & 0.10 & 0.15 & 0.20 \\
\hline 2 & 0.9048 & 9.3421 & 2.8600 & 2.0124 & 1.5709 & 1.3916 \\
\hline 4 & 0.8187 & 10.0341 & 2.9619 & 2.0646 & 1.6023 & 1.4144 \\
\hline 6 & 0.7408 & 10.7420 & 3.0618 & 2.1155 & 1.6329 & 1.4367 \\
\hline 8 & 0.6703 & 11.4590 & 3.1592 & 2.1649 & 1.6629 & 1.4584 \\
\hline 10 & 0.6065 & 12.1776 & 3.2533 & 2.2125 & 1.6919 & 1.4795 \\
\hline 15 & 0.4724 & 13.9268 & 3.4697 & 2.3223 & 1.7601 & 1.5290 \\
\hline 20 & 0.3679 & 15.4980 & 3.6522 & 2.4163 & 1.8208 & 1.5733 \\
\hline 30 & 0.2231 & 17.6479 & 3.8897 & 2.5472 & 1.9149 & 1.6434 \\
\hline 50 & 0.0821 & 17.4724 & 3.8619 & 2.5771 & 1.9826 & 1.7035 \\
\hline 100 & 0.0067 & 10.6584 & 2.8539 & 2.1016 & 1.7695 & 1.5848 \\
\hline
\end{tabular}

Table 5: Relative efficiency of PTE of $R(t)$ based on UMVUE over UMVUE of $R(t)$ for different time points $t$ and level of significance $\alpha$.

\begin{tabular}{|c|c|c|c|c|c|c|}
\hline$t$ & $\begin{array}{l}{ }^{\alpha}{ }^{\alpha} \\
R(t)^{2}\end{array}$ & 0.01 & 0.05 & 0.10 & 0.15 & 0.20 \\
\hline 2 & 0.9048 & 9.6034 & 2.9692 & 2.1070 & 1.6646 & 1.4743 \\
\hline 4 & 0.8187 & 10.2267 & 3.0528 & 2.1466 & 1.6860 & 1.4883 \\
\hline 6 & 0.7408 & 10.8683 & 3.1351 & 2.1853 & 1.7070 & 1.5019 \\
\hline 8 & 0.6703 & 11.5227 & 3.2156 & 2.2228 & 1.7273 & 1.5151 \\
\hline 10 & 0.6065 & 12.1839 & 3.2936 & 2.2590 & 1.7469 & 1.5278 \\
\hline 15 & 0.4724 & 13.8173 & 3.4743 & 2.3422 & 1.7925 & 1.5572 \\
\hline 20 & 0.3679 & 15.3180 & 3.6273 & 2.4125 & 1.8319 & 1.5825 \\
\hline 30 & 0.2231 & 17.4209 & 3.8222 & 2.5049 & 1.8879 & 1.6191 \\
\hline 50 & 0.0821 & 16.9387 & 3.7216 & 2.4745 & 1.8947 & 1.6267 \\
\hline 100 & 0.0067 & 8.3801 & 2.3738 & 1.7898 & 1.5277 & 1.3893 \\
\hline
\end{tabular}




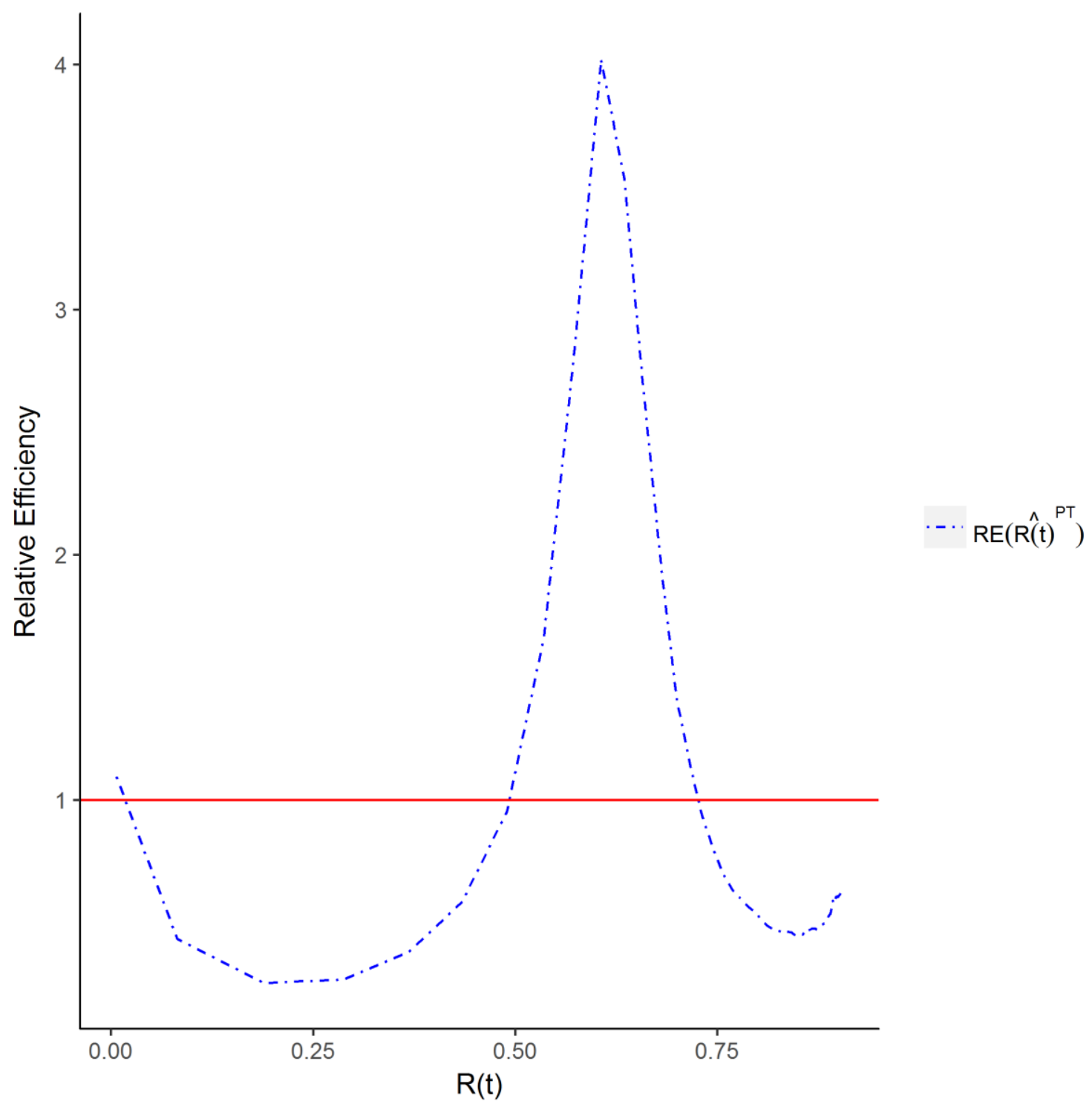

Figure 4: Relative Efficiency of $\hat{R}(t)^{P T}$ over $\widehat{R}(t)$ 


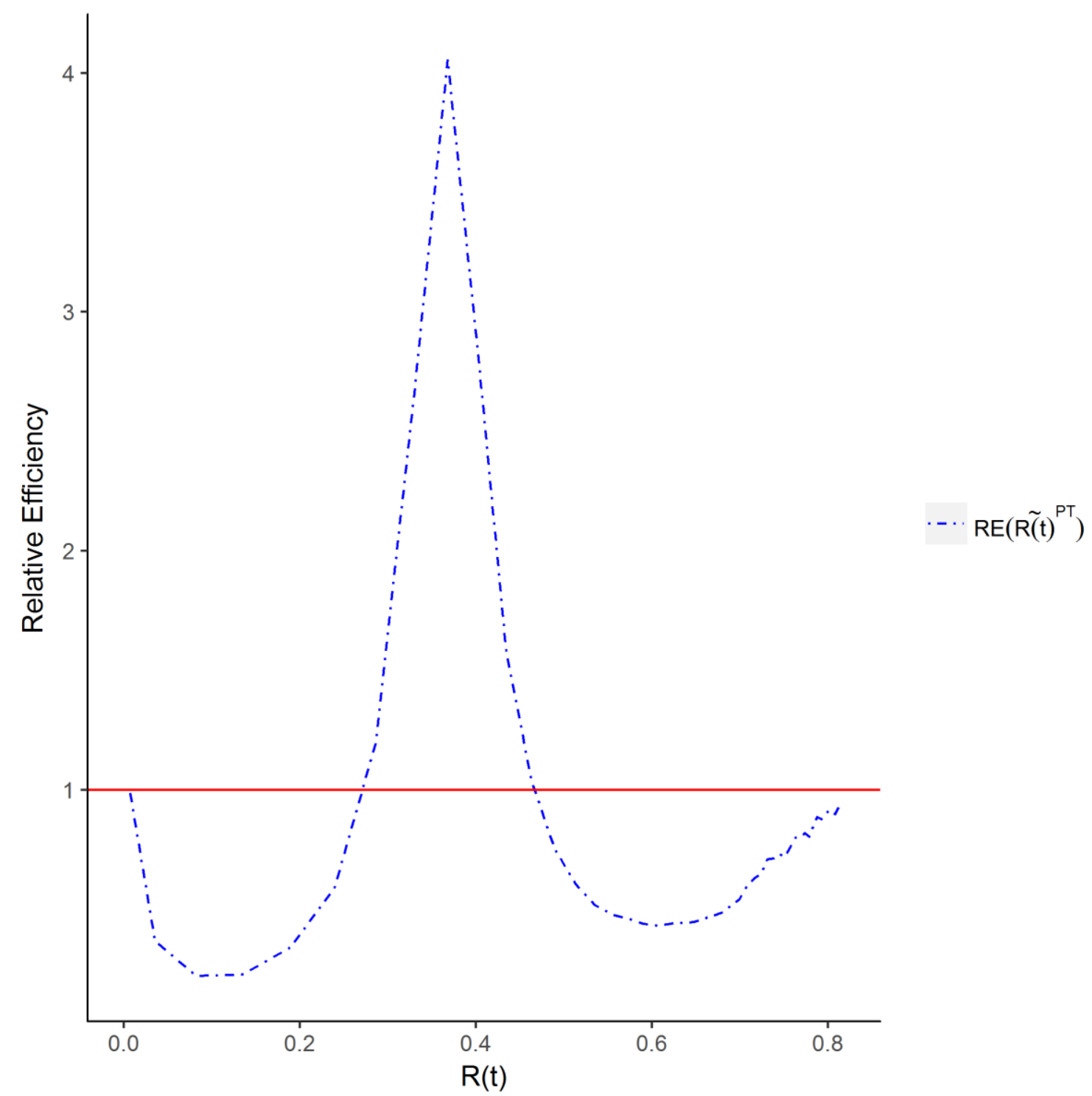

Figure 5: Relative Efficiency of $\tilde{R}(t)^{P T}$ over $\tilde{R}(t)$

Now we study the efficiency of PTES of $P$ based on MLE and UMVUE over the usual estimators of $P$ based on MLE and UMVUE. Suppose for different values of $\theta_{1}$ and $\theta_{2}$ we want to test the hypothesis $H_{o}: P=P_{o}$ against $H_{1}: P \neq P_{o}$ for fixed sample sizes $n=2$ and $m=5$. Then similar to the techniques used in Tables 3, 4 and 5, Tables 6 and 7 show the relative efficiency of PTES of $P$ based on MLE and UMVUE respectively. Figures 6 and 7 show the relative efficiency of PTES of $P$ based on MLE and UMVUE respectively with the usual estimators of $P$. From these Tables and Figures, it is clear that PTES of $P$ based on MLE and UMVUE perform better than the usual estimators of $P$ based on MLE and UMVUE respectively in the neighbourhood of the null hypothesis. 
Table 6: Relative efficiency of PTE of $P$ based on MLE over MLE of $P$ for different values of parameters and level of significance $\alpha$.

\begin{tabular}{|c|c|c|c|c|c|c|}
\hline$\theta_{1}, \theta_{2}$ & $P$ & 0.01 & 0.05 & 0.10 & 0.15 & 0.20 \\
\hline 6,5 & 0.4545 & 1.4971 & 1.3607 & 1.2470 & 1.1794 & 1.1541 \\
\hline 6,10 & 0.6250 & 1.0723 & 1.0527 & 1.0455 & 1.0388 & 1.0221 \\
\hline 6,15 & 0.7143 & 1.0129 & 1.0085 & 1.0091 & 1.0087 & 1.0026 \\
\hline 9,5 & 0.3571 & 1.0158 & 1.0146 & 1.0072 & 1.0032 & 1.0094 \\
\hline 9,10 & 0.5263 & 2.6157 & 1.9195 & 1.6185 & 1.4472 & 1.2940 \\
\hline 9,15 & 0.6250 & 1.0723 & 1.0527 & 1.0455 & 1.0388 & 1.0221 \\
\hline 12,7 & 0.3684 & 1.0246 & 1.0217 & 1.0123 & 1.0070 & 1.0130 \\
\hline 12,14 & 0.5385 & 1.8203 & 1.5336 & 1.3892 & 1.2955 & 1.1954 \\
\hline 12,21 & 0.6364 & 1.0581 & 1.0422 & 1.0370 & 1.0318 & 1.0174 \\
\hline
\end{tabular}

Table 7: Relative efficiency of PTE of $P$ based on UMVUE over UMVUE of $P$ for different values of parameters and level of significance $\alpha$.

\begin{tabular}{|c|c|c|c|c|c|c|}
\hline$\theta_{1}, \theta_{2}$ & $P$ & 0.01 & 0.05 & 0.10 & 0.15 & 0.20 \\
\hline 6,5 & 4545 & .30 & 21.3529 & 13.3 & 9.4145 & 6.5810 \\
\hline 6,10 & & 11.14 & 7.49 & 7 & 9 & 3.4372 \\
\hline 6,15 & 43 & 3.6314 & 3.2169 & 2.8315 & 2.5477 & 2.3157 \\
\hline 9,5 & 71 & 2.0522 & 1.7623 & 1.4947 & 1.2665 & 1.0325 \\
\hline 9,10 & 63 & 137.74 & 45.1773 & 26.0924 & 17.7469 & 12.0905 \\
\hline 9,15 & & 11.1402 & 7.4917 & 5.2567 & 4.1369 & 3.4372 \\
\hline 12,7 & & 2.6312 & 2.2172 & 1.8524 & 1.5517 & 1.2514 \\
\hline 12,14 & & 88.0239 & 39.1223 & 24.4535 & 17.2560 & 12.0521 \\
\hline 12,21 & 0.6364 & 9.3167 & 6.4987 & 4.7898 & 3.8645 & 3.2590 \\
\hline
\end{tabular}




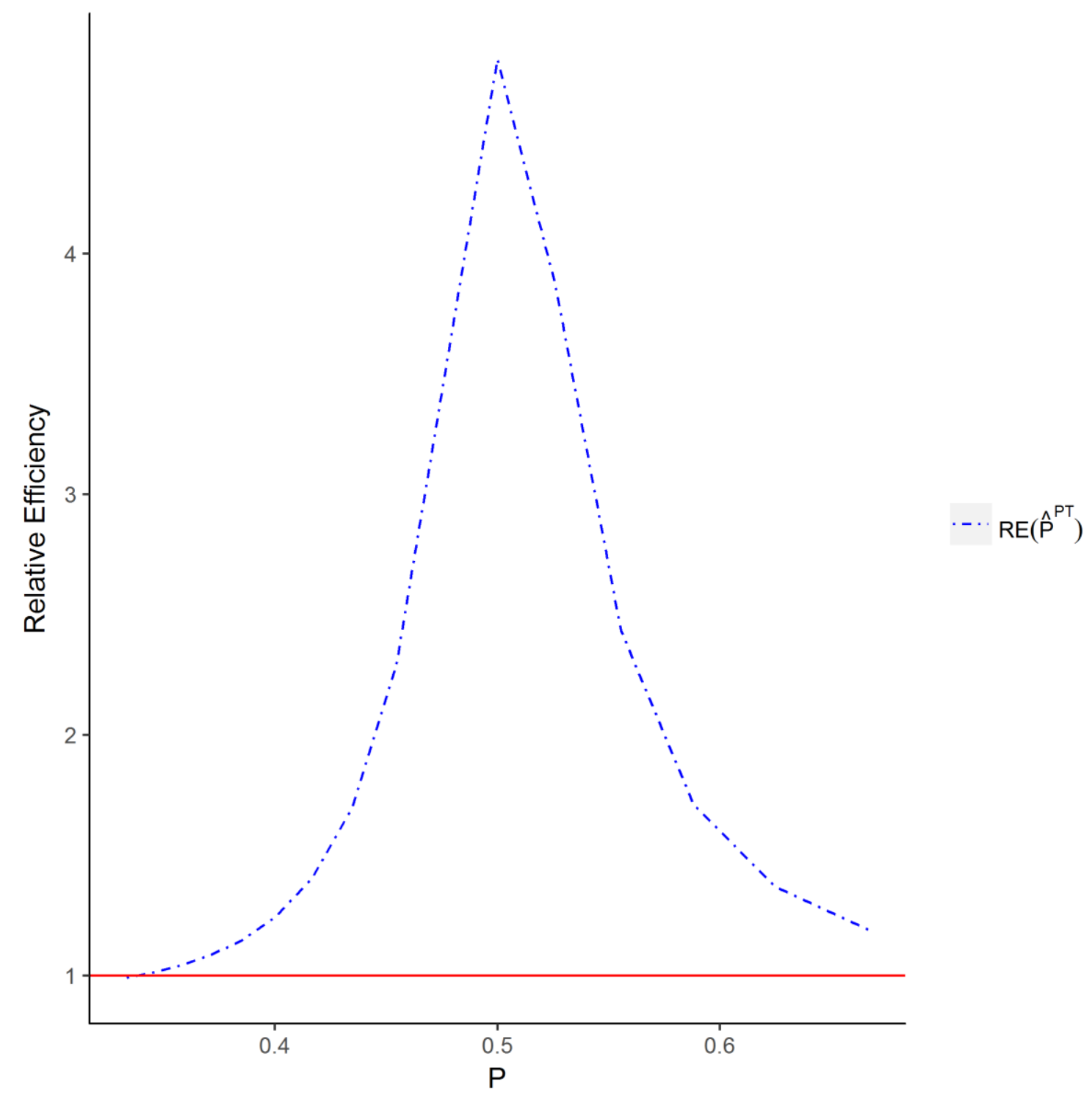

Figure 6: Relative Efficiency of $\hat{P}^{P T}$ and $\hat{P}$ 


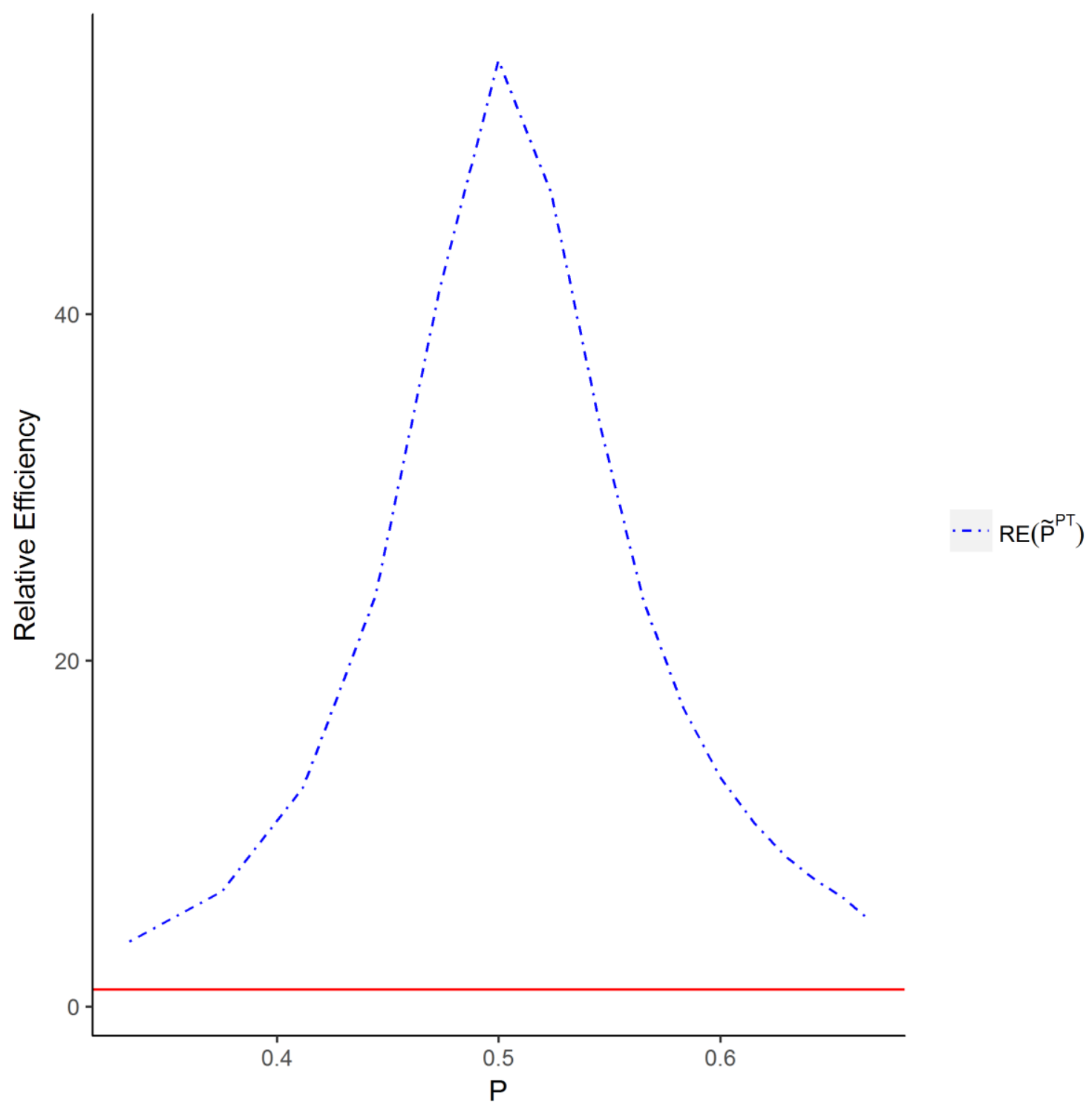

Figure 7: Relative Efficiency of $\tilde{P}^{P T}$ and $\tilde{P}$

\section{Discussion}

We developed preliminary test estimators of the powers of the parameter of exponential distribution and the reliability functions based on record data. This study is of particular interest when to estimate the reliability functions of exponential distribution; it is suspected that some uncertain prior information on the parameter of interest is available. The method involves a statistical test of the uncertain prior information and based on an appropriate statistic, a decision is made whether the sample estimate or the prior information based estimate of the parametric function should be taken.

The bias and MSE expressions of the proposed estimators are derived and along with extensive simulation procedures, we compare the performance of the PTES over the usual 
estimators. It can be concluded that all of the proposed PTES of the powers of the parameter of the exponential distribution and its reliability function dominate their corresponding usual estimators such as UMVUE, MLE and EBE in the sense that they exhibit a lower mean square error whenever the true value of the parameter is close to the hypothesised value. However the PTES perform much worse when the true value is far away from the hypothesised value of the parametric functions.

\section{Acknowledgement}

We are grateful to the Editor and the Reviewers for their fruitful suggestions which lead to a considerable improvement in this research paper.

\section{References}

[1] Arnold, B. C., Balakrishan, N. and Nagaraja, H. N. (1992): A First Course in Order Statistics. John Wiley \& Sons, New York.

[2] Arashi, M. and Emadi, M. (2008): Evidential inference based on record data and inter-record times. Statist. Papers, 13(8), 380-210.

[3] Awad, A. M. and Gharraf, M. K. (1986): Estimation of $\mathrm{P}(\mathrm{Y}<\mathrm{X})$ in the Burr case: A Comparative Study. Commun. Statist. - Simul., 15(2), 389-403.

[4] Balakrishan, N., Ahsanullah, M. And Chan, P. S. (1995): On the logistic record values and associated inference. Jour. Appl. Statist. Scien., 2, 233-248.

[5] Bartholomew, D. J. (1957): A problem in life testing. Jour. Amer. Statist. Assoc., 52, 350355.

[6] Bartholomew, D. J. (1963): The sampling distribution of an estimate arising in life testing. Technometrics, 5, 361-374.

[7] Basu, A. P. (1964): Estimates of reliability for some distributions useful in life testing. Technometrics, 6, 215-219.

[8] Belaghi, R. A., Arashi, M., Tabatabaey, S. M. M. (2014): Improved confidence intervals for the scale parameter of Burr XII model based on record values. Comput. Stat., DOI 10.1007/s00180-014-0484-3.

[9] Belaghi, R. A., Arashi, M. and Tabatabaey, S. M. M. (2015): On the Construction of Preliminary Test Estimator Based on Record Values for the Burr XII Model. Commun. Statist. - Theo. Meth., 44(1), 1-23, DOI:10.1080/03610926.2012.733473

[10] Chandler, K. N. (1952): The distribution and frequency of record values. Jour. Royal Statist. Socie., Series B, 14, 220-228. 
[11] Chao, A. (1982): On comparing estimators of $\operatorname{Pr}\{\mathrm{X}>\mathrm{Y}\}$ in the exponential case. IEEE Trans. Reliability, R-26, 389-392.

[12] Chaturvedi, A. and Kumari, T. (2015): Estimation and testing procedures for the reliability functions of a family of lifetime distributions. interstat.statjournals.net/ YEAR/2015/ abstracts/ 1306001.php

[13] Chaturvedi, A. and Malhotra, A. (2016): Estimation and Testing Procedures for the Reliability Functions of a Family of Lifetime Distributions based on Records. Int. J. Syst. Assur. Eng. Manag., DOI:10.1007/s13198-016-0531-2

[14] Chaturvedi, A. and Malhotra, A. (2017): Inference on the Parameters and Reliability Characteristics of three parameter Burr Distribution based on Records. Appl. Math. Inf. Scien., 11(3), 837-849.

[15] Chaturvedi, A. and Pathak, A. (2012): Estimation of the reliability functions for exponentiated Weibull distribution. J. Stat. \& Appl, 7, 1-8.

[16] Chaturvedi, A. and Pathak, A. (2013): Bayesian estimation procedures for three parameter exponentiated Weibull distribution under entropy loss function and type II censoring. interstat.statjournals.net/YEAR/2013/abstracts/1306001.php

[17] Chaturvedi, A. and Pathak, A. (2014): Estimation of the Reliability Function for fourParameter Exponentiated Generalized Lomax Distribution. IJSER, 5(1), 1171-1180.

[18] Chaturvedi, A. and Rani, U. (1997): Estimation procedures for a family of density functions representing various life-testing models. Metrika, 46, 213-219.

[19] Chaturvedi, A. and Rani, U. (1998): Classical and Bayesian reliability estimation of the generalized Maxwell failure distribution. Jour. Statist. Res., 32, 113-120.

[20] Chaturvedi, A. and Singh, K. G. (2006): Bayesian estimation procedures for a family of lifetime distributions under squared-error and entropy losses. Metron, 64(2), 179-198.

[21] Chaturvedi, A. and Singh, K. G. (2008): A family of lifetime distributions and related estimation and testing procedures for the reliability function. Jour. Appl. Statist. Sci., 16(2), 35-50.

[22] Chaturvedi, A. and Surinder, K. (1999): Further remarks on estimating the reliability function of exponential distribution under type I and type II censorings. Brazi. Jour. Prob. Statist., 13, 29-39.

[23] Chaturvedi, A. and Tomer, S. K. (2002): Classical and Bayesian reliability estimation of the negative binomial distribution. Jour. Appl. Statist. Scien., 11, 33-43. 
[24] Chaturvedi, A. and Tomer, S. K. (2003): UMVU estimation of the reliability function of the generalized life distributions. Statist. Papers, 44(3), 301-313.

[25] Constantine, K., Karson, M. and Tse, S. K. (1986): Estimation of $\mathrm{P}(\mathrm{Y}<\mathrm{X})$ in the gamma case. Commun. Statist. - Simul., 15(2), 365-388.

[26] Epstein, B. and Sobel, M. (1953): Life Testing. Jour. Amer. Statist. Assoc., 48, 486-502.

[27] Glick, N. (1978): Breaking records and breaking boards. Amer. Math. Month., 85, 543-551.

[28] Habibi Rad, A., Arghami, N. R. and Ahmadi, J. (2006): Statistical evidence in experiments and in record values. Commun. Stat. - Theo. Meth. 35(11), 1971-1983.

[29] Johnson, N. L. (1975): Letter to the editor. Technometrics, 17, 393.

[30] Kelly, G. D., Kelly, J. A. And Schucany, W. R. (1976): Efficient estimation of $\mathrm{P}(\mathrm{Y}<\mathrm{X})$ in the exponential case. Technometrics, 18, 359-360.

[31] Kibria, B. M. G. (2004): Performance of the shrinkage preliminary tests ridge regression estimators based on the conflicting of W, LR and LM tests. Jour. Statist. Comp. Simul., 74(11), 793-810.

[32] Kibria, B. M. G. and Saleh, A. K. M. E. (1993): Performance of shrinkage preliminary test estimator in regression analysis. Jahangrinagar Rev., A17, 133-148.

[33] Kibria, B. M. G. and Saleh, A. K. M. E. (2004): Preliminary test ridge regression estimators with Student's t errors and conflicting test-statistics. Metrika., 59(2), 105-124.

[34] Kibria, B. M. G. and Saleh, A. K. M. E. (2005): Comparison between Han-Bancroft and Brook method to determine the optimum significance level for pre-test estimator. Jour. Prob. Statist. Scien., 3, 293-303.

[35] Kibria, B. M. G. and Saleh, A. K. M. E. (2006): Optimum critical value for pre-test estimators. Comm. Statist.- Theo. Meth., 35(2), 309-320.

[36] Kibria, B. M. G. and Saleh, A. K. M. E. (2010): Preliminary test estimation of the parameters of exponential and Pareto distributions for censored samples. Statist. Papers., 51, 757-773.

[37] Kotz, S., Lumelskii, Y. and Pensky, M. (2003): The Stress-Strength Model and its Generalizations, Theory and Applications. World Scientific.

[38] Nagaraja, H. N. (1988a): Record values and related statistics - A review. Commun. Statist. Theo. Meth., 17, 2223-2238.

[39] Nagaraja, H. N. (1988b): Some characterizations of continuous distributions based on regressions of adjacent order statistics and record values. Sankhya, Series A, 50, 70-73.

[40] Pugh, E. L. (1963): The best estimate of reliability in the exponential case. Operations Research, 11, 57-61. 
[41] Razmkhah, M. and Ahmadi, J. (2011): Comparing two sampling schemes based on entropy of record statistics. Statist. Papers, 53, 95-106.

[42] Saleh, A. K. M. E. and Kibria, B. M. G. (1993): Performance of some new preliminary test ridge regression estimators and their properties. Commum. Statist. - Theo. Meth., 22(10), 2747-2764.

[43] Saleh, A. K. M. E. and Sen, P. K. (1978): Nonparametric estimation of location parameter after a preliminary test on regression. Ann. Statist., 6, 154-168.

[44] Sinha, S. K. (1986): Reliability and Life Testing. Wiley Eastern Limited, New Delhi.

[45] Tong, H. (1974): A note on the estimation of $\mathrm{P}(\mathrm{Y}<\mathrm{X})$ in the exponential case. Technometrics, 16, 625.

[46] Tong, H. (1975): Letter to the editor. Technometrics, 17, 393.

[47] Tyagi, R. K. and Bhattacharya, S. K.(1989): A note on the MVU estimation of reliability for the Maxwell failure distribution. Estadistica, 41, 73-79.

[48] Watson, R. I. (1952): Research design and methodology in evaluating the results of psychotherapy, J. Clin. Psychol., 8, 29-33. DOI:10.1002/1097-4679(195201)8:1<29::AIDJCLP2270080107>3.0.CO;2-O 